\title{
The role of nurses in the management of heart failure
}

\section{J Grange}

Heart 2005;91(Suppl II):ii39-ii42. doi: 10.1136/hrt.2005.062117

Care provided by specialist nurses has been shown to improve outcomes for patients with chronic heart failure (CHF), significantly reducing the number of unplanned readmissions, length of hospital stay, hospital costs, and mortality. Most patients develop CHF as a result of coronary artery disease. Once cardiac damage has occurred, the risk of developing heart failure can be reduced by providing appropriate treatment at appropriate dosages. While cardiac rehabilitation clinics provide an opportunity to check drug usage, their prime focus is on optimising patients' physical well being following a heart attack. In addition, evidence suggests that general practitioners are frequently reluctant to initiate appropriate treatments and to up-titrate drug dosages even for patients with diagnosed heart failure. Therefore, to ensure that these patients are not left on starting doses of medications many hospitals are now setting up nurse led post-myocardial infarction (MI) clinics. The Omada programme is a secondary care based, nurse led model of care set up in 1999 to improve the management of CHF by providing appropriate patient education within a nurse led clinic setting, optimising evidence based medication and fostering partnership between health professionals in both primary and secondary care. The model of care is highly applicable to the post-Ml setting, where it can ensure that patients receive better care at an earlier stage.

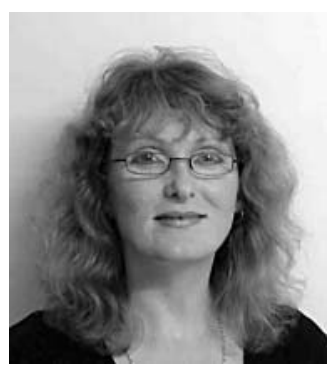

Correspondence to: Julie Grange, Ashfield Healthcare Ltd, Ashfield House, Resolution Road, Ashby-de-la-Zouch, Leicestershire, LE65 1HW, UK; igrange@ ashfieldhealthcare.com

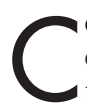
oronary heart disease (CHD) is the most common cause of premature death before the age of 75 years in the UK. It is also the most common cause of heart failure in western countries, responsible for an estimated $70 \%$ of heart failure cases in the UK. Furthermore, the UK has one of the highest death rates from CHD; only Ireland and Finland have higher rates.

However, death rates from CHD have been falling since the late 1970s; in the past 10 years, there has been a $44 \%$ reduction among those under 65 years of age. These improvements are said to be the result of favourable trends in risk factors, as well as the improved uptake of effective treatments.

While this is a welcome trend, there is still a long way to go. It is estimated that around $40 \%$ of myocardial infarctions are accompanied by left ventricular dysfunction, with or without clinical heart failure. ${ }^{2}$ Approximately $22 \%$ of male and $46 \%$ of female myocardial infarction (MI) sufferers will be disabled with heart failure within six years. ${ }^{3}$ Although the modern management of MI is improving the survival of patients, it is leaving them with a significantly compromised heart that is likely to fail at some time in the future.

It is estimated that $4 \%$ of men and $2 \%$ of women have had an MI; some 826000 people under the age of 75 years living in the UK have had an MI. However, the prevalence of MI increases with age, suggesting that some 1.2 million people living in the UK have had an MI. If a high proportion of these are liable to develop heart failure within six years, then it is important that we recognise these patients on the ward and ensure that we have the resources to manage this rapidly growing group of patients.

Once cardiac damage has occurred, typically from a heart attack, the risk of developing heart failure can be reduced by providing appropriate treatment at appropriate dosages. While cardiac rehabilitation clinics provide an opportunity to check drug usage, their prime focus is on optimising patients' physical wellbeing following a heart attack. Therefore, to ensure that these patients are not left on starting doses of medications, many hospitals are now setting up nurse led post-MI clinics, building on the models successfully developed for managing patients with chronic heart failure $(\mathrm{CHF})$.

\section{MANAGEMENT OF CHF}

CHF is a complex syndrome that commonly affects elderly patients in whom it has a major impact upon longevity and quality of life. It is usually associated with symptoms such as dyspnoea, fatigue, and fluid retention, and results in frequent episodes of hospitalisation. Numerous clinical trials have shown that symptoms can be managed by drug treatments and modification of various lifestyle factors, resulting in reduced hospitalisation and improvements in morbidity and mortality. However, both patient characteristics and shortfalls in care delivery mean that the benefits seen in trials are frequently not observed in clinical practice. This, in part, is due to the fact that evidence based treatments are often not titrated to optimal levels due to lack of expertise, lack of time, difficulty in diagnosis, co-morbid conditions, and poor coordination of care.

Heart failure patients frequently have coexisting conditions, for which they take many medications. Often they may have cognitive and/ or functional limitations, and may be isolated with poor social support. ${ }^{4}$ Many will have financial concerns, and psychological conditions

Abbreviations: CHD, coronary heart disease; CHF, chronic heart failure; MI, myocardial infarction 


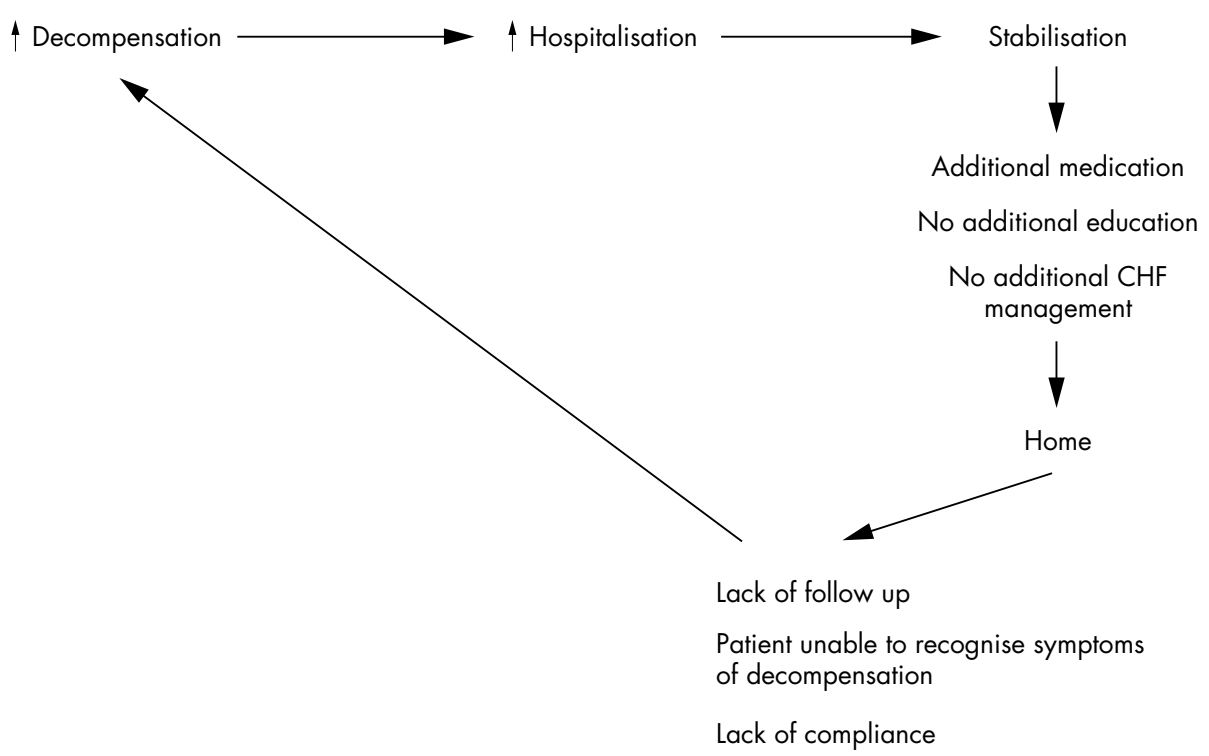

Figure 1 The vicious cycle of repeat hospitalisation that may occur with the traditional approach to heart failure management.

Additional medication

aducation

additional $\mathrm{CHF}$

such as anxiety and depression are common. Heart failure patients frequently have inadequate access to health care personnel, receive poor follow up, and are poor compliers with medications.

From the patient's perspective, the disease is characterised by a vicious cycle of worsening symptoms, acute decompensation, hospitalisation, and subsequent stabilisation and discharge (fig 1). However, because patients often feel substantially better after hospitalisation, they are liable to feel they do not need to take so many tablets. Poor compliance with medication means the patient is more likely to decompensate, leading to re-hospitalisation and the need to re-stabilise the patient.

However, this vicious cycle can be broken by instituting a structured system of care delivery involving a multidisciplinary team that provides the patient with education about their condition, medications, and when to seek help for worsening symptoms (fig 2). ${ }^{4}$

It is estimated that $64 \%$ of re-admissions are caused by non-compliance ${ }^{5}$ while $54 \%$ of re-admissions are preventable. ${ }^{6}$ Inadequate discharge planning or follow up is a common factor in readmission.
THE CASE FOR SPECIALISED STRUCTURED CARE IN CHF

In the early 1980s it was recognised that a nurse led heart failure service can bring about significant reduction in rehospitalisation and total days spent in hospital. ${ }^{7}$

Subsequent studies demonstrated that intensive homecare programmes delivered by nurses were associated with a notable decrease in the need for hospitalisation and improved the functional status of elderly patients with severe $\mathrm{CHF}^{8}$; moreover, among a cohort of high risk patients with CHF, home based intervention was associated with reduced frequency of unplanned readmissions plus out-of-hospital deaths within six months of discharge from hospital. ${ }^{9}$

Care provided by specialist nurses has been shown to improve outcomes for patients with CHF, significantly reducing the number of unplanned readmissions, length of hospital stay, mortality, and hospital costs..$^{11}$

Furthermore, patients with CHF have fewer hospitalisations for heart failure and are significantly more active when managed by heart failure specialists working in a dedicated heart failure programme rather than by physicians with limited expertise in heart failure. ${ }^{12}$

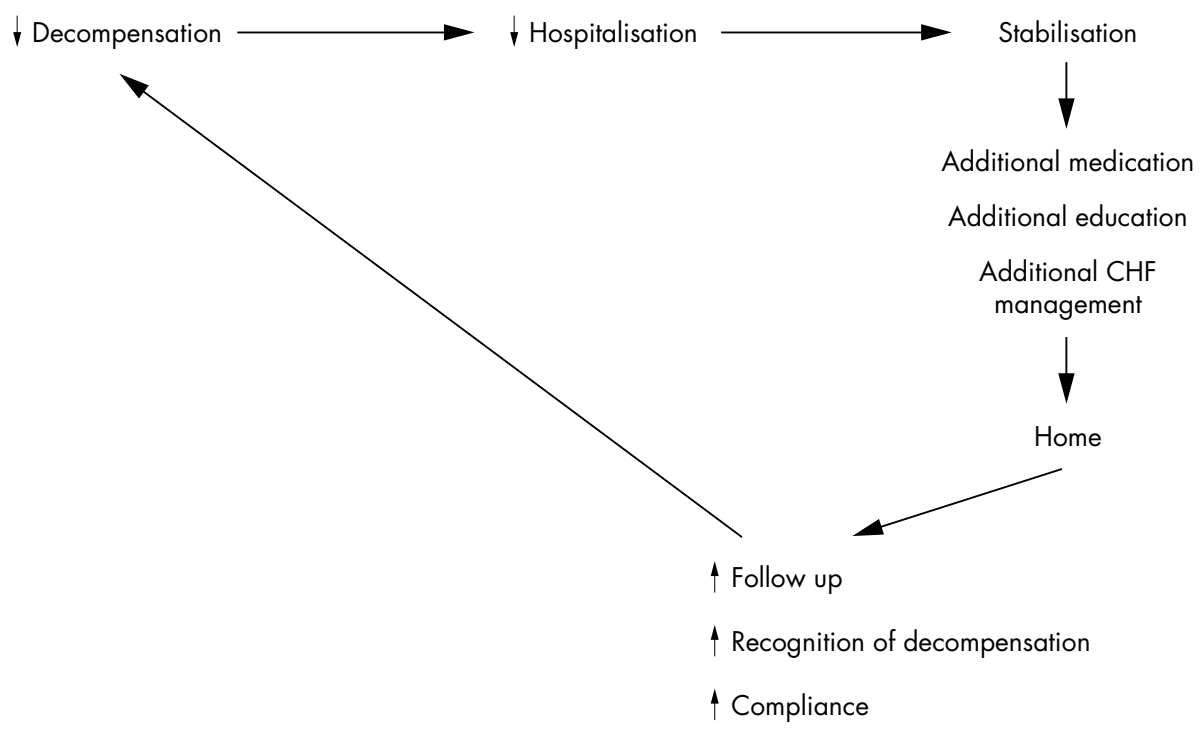

Figure 2 How specialist intervention can break the vicious cycle of repeat hospitalisation.

Compliance 
Yet despite such evidence, even today there are still many centres around the UK that do not have a heart failure service, a situation that has prompted government action. The National Service Framework for CHD stated that doctors should arrange for people with suspected heart failure to be offered appropriate investigations (for example, electrocardiography, echocardiography) that will confirm or refute the diagnosis. For those in whom heart failure is confirmed, its cause should be identified - the treatments most likely to both relieve symptoms and reduce their risk of death should be offered. This was to be demonstrated by clinical audit data no more than 12 months old. ${ }^{13}{ }^{14}$ In achieving this aim, the National Service Framework envisaged:

- specialist heart failure nurses

- nurse led heart failure clinics

- outreach and community services.

It is now widely accepted that a specialist heart failure nurse working under medical supervision can make a major difference to meeting the many needs of these patients (fig 3 ). Specialist nurses have a wealth of knowledge and expertise in patient management and support. Instead of a standard 10 minute consultation, a nurse can spend time with patients and relatives, dealing with their worries and concerns and providing support and understanding when it is needed. This leads to increased patient satisfaction and, as problems are addressed, to improved quality of life.

Management of heart failure encompasses both pharmacological and non-pharmacological approaches. The nurse can play an important role in initiating and up-titrating appropriate medications. Most patients will have a variety of co-morbid conditions for which they are likely to be receiving many medications. Against this background, it takes time, knowledge and expertise to ensure that patients' medications are safely optimised, time which most general practitioners and consultants are unable to give.

Providing education is crucial. Patients with heart failure need to understand their condition, how to manage it, and above all when to ask for assistance. Moving away from the traditional view of the patient as a passive recipient of information towards being an active partner in the management of their health can help empower them. Most patients are able, after education, to manage their own diuretic medication, monitoring their weight and tailoring diuretic dosages appropriately, provided they know when to contact a nurse or doctor for advice.

\section{THE OMADA CHF PROGRAMME}

The Omada programme is a secondary care based, nurse led model of care that was established in 1999 to improve the management of CHF by providing appropriate patient education within a structured nurse led clinic setting, optimising evidence based medication and fostering partnership between health professionals in both primary and secondary care. ${ }^{15}$

Initially, a team of trained cardiac nurses were employed with an educational grant from a pharmaceutical company to assess the shortfalls in the management of heart failure across the UK and to help optimise care for heart failure patients.

An initial audit within nine centres across the UK identified a cohort of patients who required optimisation of medical treatments. Using nationally and locally agreed protocols, clinics were set up within secondary care departments with a dedicated nurse running clinics under medical supervision. Patients were invited to attend and their needs were assessed with regards to education, and initiation and optimisation of medications. Strong clinical links were formed within primary and secondary care.

Subsequent audit of the programme within the nine centres has demonstrated improved levels of care and increased uptake of evidence based treatments for patients with CHF. ${ }^{15}$ The knowledge and experienced gained through the programme is now available to assist other centres in setting up and running their own services.

The heart failure clinic is based on five key principles:

- multidisciplinary approach

- treatment specifically for heart failure patients

- continuous, long term patient support (medical, social, psychological)

- education for patients and relatives

- inclusion of non-pharmacological as well as drug treatment.

In addition, the programme facilitates the transfer of information from secondary to primary care, along with the rationale for the management plan.

\section{OMADA AND POST-MI HEART FAILURE CARE}

Although set up initially to help patients with CHF, the focus of the Omada programme has now shifted to looking at how to help health care professionals address the problem of

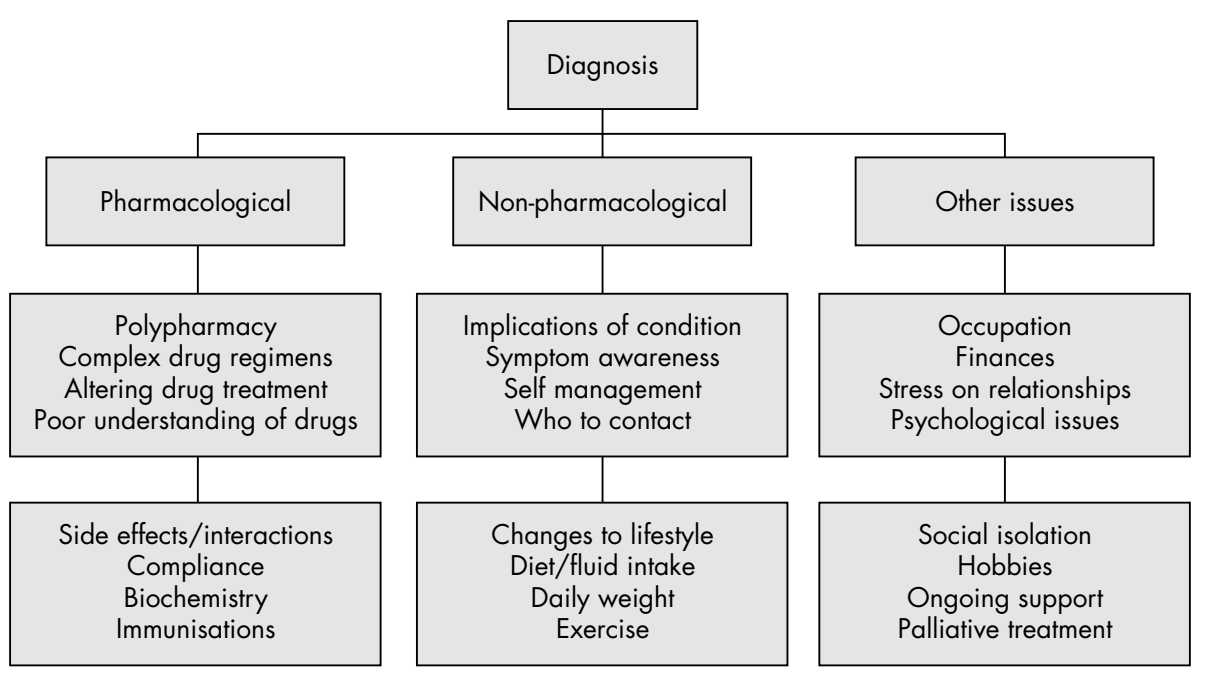

Figure 3 The multiple needs of a heart failure patient following diagnosis. 
Table 1 How a heart failure clinic fits into a typical postMI treatment pathway

\begin{tabular}{lll}
\hline Day 1-3 & CCU & $\begin{array}{l}\text { Initiation of drug treatment for disease } \\
\text { modification and symptom control }\end{array}$ \\
Day 3-10 & $\begin{array}{l}\text { General medical } \\
\text { ward }\end{array}$ & $\begin{array}{l}\text { Treatment optimised for discharge; } \\
\text { general rehabilitation }\end{array}$ \\
Week 6 & $\begin{array}{l}\text { Cardiology clinic } \\
\text { Ongoing cardiac rehabilitation; seen } \\
\text { by cardiologist }\end{array}$ \\
Week 8+ & $\begin{array}{l}\text { Nurse run HF } \\
\text { clinic }\end{array}$ & $\begin{array}{ll}\text { Lifestyle evaluation; education/support } \\
\text { Month 6 }\end{array}$ \\
\hline
\end{tabular}

$\mathrm{HF}$, heart failure.

post-MI heart failure, by promoting evidence based practice, educational sessions, and care pathway documentation.

If no integrated care pathways are in place then patients can easily slip through the care net without receiving good evidence based treatment and management. In order to enable nurses to improve standards of care throughout their centres, the Omada programme has now developed three core integrated care pathways, designed to address:

- post-MI management

- clinical signs of acute heart failure

- CHF.

In addition, there is a proforma for post-MI audit.

Following an MI, patients with signs of heart failure may access the nurse led clinic through a variety of means depending on local pathways. These include:

- during inpatient stay

- referral from cardiology outpatient clinics

- referral from cardiac rehabilitation

- referral from other outpatient clinics

- referral from general practice.

Table 1 shows how a heart failure clinic fits into a typical post-MI treatment pathway.

\section{CONCLUSION}

Structured programmes of heart failure care result in significant benefits to patients in terms of:

- improved quality of life

- improved education

- individualised care

- appropriate medication.

In addition, there are benefits to the hospital in terms of decreased admissions, reduction in "bed blocking", and compliance with clinical governance objectives and national targets.

By ensuring that patients receive optimal evidence based treatment at the right time, and that progress is monitored, such programmes can help to break the vicious cycle of suboptimal heart failure care. By focusing on education, not just of the patients but also of their relatives and the nurses' hospital colleagues and other health care professionals, programmes such as Omada have made a great difference
Learning points

- Early identification of patients with post-myocardial infarction heart failure allows the opportunity to improve their quality of life, prolong their life, and keep them out of hospital

- Nurse led clinics are ideally placed to empower patients by giving them the education and tools they need to deal with their condition

- A systematic, protocol driven approach can optimise the uptake of evidence based drug treatments.

- The Omada nurses offer a useful resource to others interested in setting up nurse led clinics.

to the management of CHF. The Omada programme offers a useful resource to others interested in setting up nurse led clinics both in CHF and also in the setting of post-MI heart failure.

Julie Grange is an employee of Ashfield Healthcare working on the Omada project. Omada is sponsored by an unrestricted educational grant from Pfizer.

\section{REFERENCES}

1 British Heart Foundation. Heartstats, 2004. London: BHF, 2005.

2 Kober L, Torp-Pedersen C, Jorgensen S, et al. Changes in absolute and relative importance in the prognostic value of left ventricular systolic function and congestive heart failure after acute myocardial infarction. TRACE study group. Trandolapril cardiac evaluation. Am J Cardiol 1998;81:1292-7.

3 American Heart Association. Heart disease and stroke statistics - 2003 update. Atlanta, Georgia: AHA, 2002:120.

4 Moser DK. In: Reiger B, ed. Improving outcomes in heart failure: an interdisciplinary approach. Gaithersburg: Aspen Publishers Incorporated, 2001.

5 Ghali JK, Kadakia S, Cooper R, et al. Precipitating factors leading to decompensation of heart failure. Traits among urban blacks. Arch Intern Med 1988;148:2013-6.

6 Michalsen A, Konig G, Thimme W. Preventable causative factors leading to hospital admission with decompensated heart failure. Heart 1998;80:437-41.

7 Cintron G, Bigas C, Linares $E$, et al. Nurse practitioner role in a chronic congestive heart failure clinic: in-hospital time, costs, and patient satisfaction. Heart Lung 1983;12:237-40.

8 Kornowski R, Zeeli D, Averbuch $M$, et al. Intensive home-care surveillance prevents hospitalization and improves morbidity rates among elderly patients with severe congestive heart failure. Am Heart J 1995;129:762-6.

9 Stewart S, Pearson S, Horowitz JD. Effects of a home-based intervention among patients with congestive heart failure discharged from acute hospital care. Arch Intern Med 1998;158:1067-72.

10 Blue L, Lang E, McMurray JJV, et al. Randomised controlled trial of specialist nurse intervention in heart failure. BMJ 2001;323:715-8.

11 Stewart S, Vandenbroek AJ, Pearson S, et al. Prolonged beneficial effects of a home-based intervention on unplanned readmissions and mortality among patients with congestive heart failure. Arch Intern Med 1999;159:257-61.

12 Hanumanthu S, Butler J, Chomsky D, et al. Effect of a heart failure program on hospitalization frequency and exercise tolerance. Circulation 1997:96:2842-8.

13 Department of Health. National service framework for coronary heart disease. London: Department of Health, 2000, http://www.dh.gov.uk/ PolicyAndGuidance/HealthAndSocialCareTopics/CoronaryHeartDisease/ fs/en.

14 Department of Health. Developing services for heart failure 2003. London: Department of Health. hitp://www.dh.gov.uk/PolicyAndGuidance/ HealthAndSocialCareTopics/CoronaryHeartDisease/fs/en.

15 Cowie MR, Mclntyre H, Panahloo Z. Delivering evidence-based care to patients with heart failure: results of a structured programme in nine UK centres. Br J Cardiol 2002;9:171-80. 